\title{
HACER EL CRETENSE PROVERBIALMENTE
}

\author{
Fernando García Romero \\ Universidad Complutense de Madrid \\ fgarciar@ucm.es
}

\section{RESUMEN}

Se analizan locuciones y expresiones proverbiales griegas antiguas que tienen como protagonistas a los cretenses, de los cuales ofrecen generalmente una imagen negativa.

PALABRAS ClaVE: proverbio, Creta, Grecia antigua.

PLAY THE CRETAN PROVERBIALLY

\section{ABSTRACT}

Ancient Greek proverbial expressions are analyzed, that have the Cretans as protagonists; usually a negative image is offered.

KeYWORDS: proverbs, Crete, ancient Greece.

Ángel Martínez Fernández es sin duda un sujeto gramatical adecuadísimo

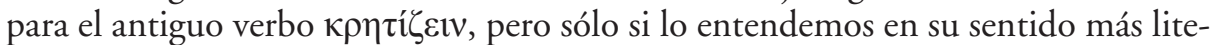
ral de 'hacer el cretense, comportarse como un cretense' e incluso 'hablar cretense' (Amado Rodríguez, 1995: 85). El sujeto ha de ser otro, desde luego, si entendemos el verbo en su sentido connotativo y negativo, el más habitual con diferencia en los textos que han llegado hasta nosotros, hasta el punto de haberse hecho proverbial y aparecer recogido en las compilaciones lexicográficas y en varias de las colecciones paremiográficas que forman el Corpus Paroemiographorum Graecorum, por

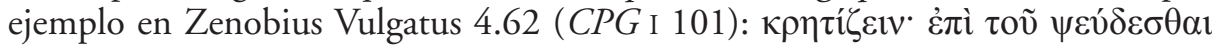

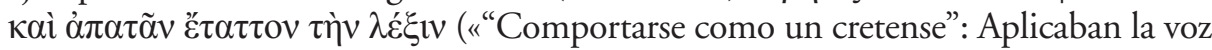
al hecho de mentir y engañar») ${ }^{1}$.

La glosa de la colección Zenobius Vulgatus busca en el mito el origen de ese

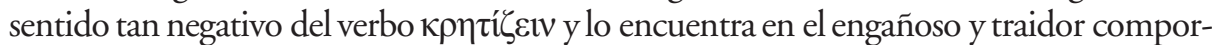
tamiento de Idomeneo, el caudillo homérico de los cretenses, en una ocasión en la que

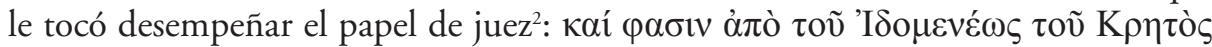

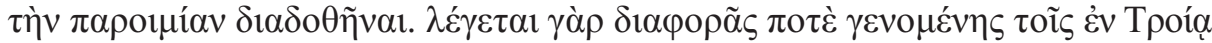
"E

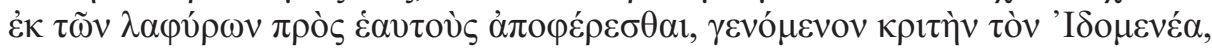




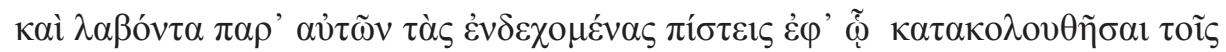

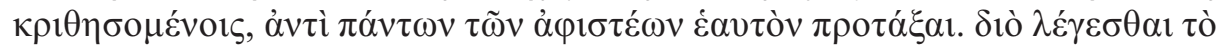
$\kappa \rho \eta \tau i ́ \zeta \varepsilon ı$ ( Y afirman que el proverbio se difundió a partir del cretense Idomeneo. Porque se dice que hubo en cierta ocasión un desacuerdo entre los griegos de Troya sobre quién era más importante, y como todos deseaban llevarse para sí mismos el bronce recogido de los despojos de guerra, fue juez Idomeneo y, después de tomar de ellos los juramentos de conformidad con obedecer lo que se fuera a decidir, se lo asignó a sí mismo en lugar de a cualquiera de los más distinguidos. Por eso se decía "comportarse como un cretense"»). Otras fuentes (Suda к 2407 Adler, escolios al Himno

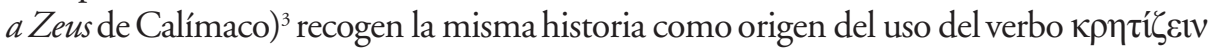
en el sentido de 'engañar traicioneramente', en tanto que una noticia transmitida por Focio (Biblioteca 190, 150a38-150b3 Henry) atribuye también a Idomeneo el hecho de que el 'comportamiento cretense' sea sinónimo de engaño, pero cambia

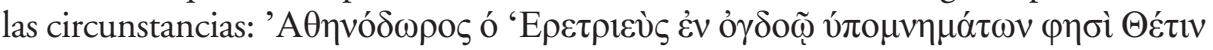

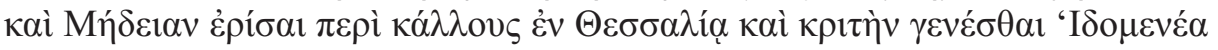

${ }^{1}$ Véanse también, con explicaciones similares, Diogeniano 5.58 (CPGI 262), Gregorio de Chipre Mosquensis 3.87 (CPG II 119), Apostolio 10.7 (CPG II 487) y, entre los lexicógrafos, Hesiquio $\kappa 4086$ Latte y Suda $\kappa 2407$ Adler. En la colección Zenobius Athous 3.54 (editada por Miller, 1868; la misma glosa en Collectio Bodleiana 860, recogida en la Appendix Proverbiorum del CPG, I 452) el verbo $\kappa \rho \eta \tau i \zeta \xi ı v$ aparece, sin explicación de sentido y origen, junto con otros dos verbos denominativos forma-

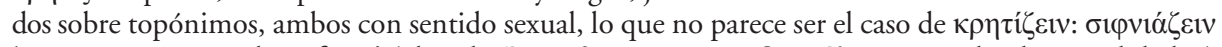
'comportarse como los sifnios' (glosado ä $\pi \tau \varepsilon \sigma \theta \alpha \imath \tau \tilde{\eta} \varsigma \pi v \gamma \tilde{\eta} \varsigma \delta \alpha \kappa \tau \dot{\nu} \lambda \omega$ «tocar el culo con el dedo») y $\lambda \varepsilon \sigma \beta i \alpha ́ \zeta \varepsilon ı v$ 'comportarse como los lesbios' (glosado $\pi \alpha \rho \alpha v o \mu \varepsilon \tau i v \sigma \tau o ́ \mu \alpha \tau 1$ «realizar perversiones

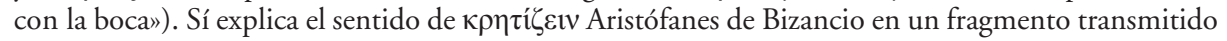

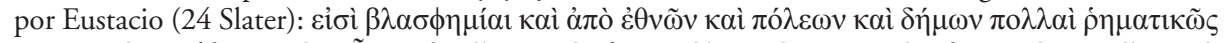

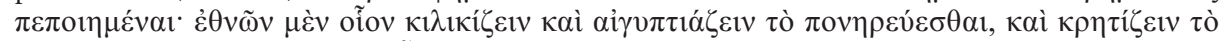

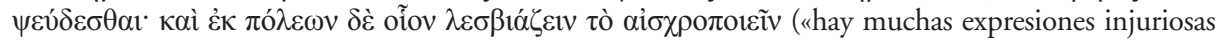
construidas en forma de verbo a partir de etnias, ciudades y pueblos: de etnias, por ejemplo, 'comportarse como los cilicios' y 'comportarse como los egipcios' en el sentido de 'ser malvado', y 'comportarse como los cretenses' en el sentido de 'mentir'; y a partir de nombres de ciudades, por ejemplo, 'comportarse como los lesbios' en el sentido de 'comportarse obscenamente'»). Las colecciones Zenobius Vulgatus y Zenobius Athous están traducidas al español por Mariño Sánchez-Elvira - García Romero (1999).

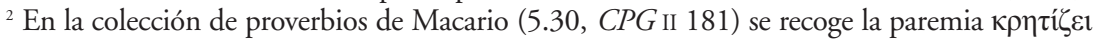

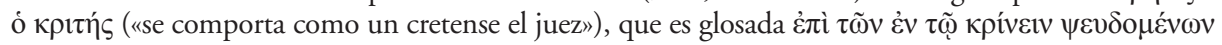
(«a propósito de los que mienten cuando juzgan»).

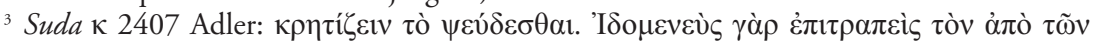

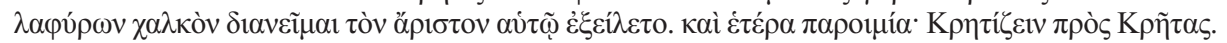

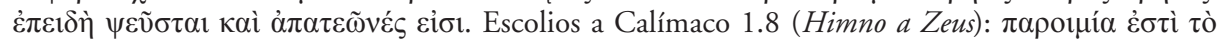

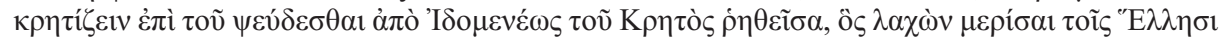

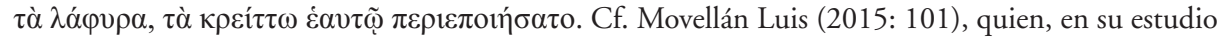
de la crónica de la Guerra de Troya atribuida al cretense Dictis, sugiere que «es posible que la aparición aquí de Idomeneo como buen consejero a la hora de hacer el reparto [del botín] sea un intento por parte del autor de la Ephemeris de rebatir la tradición proverbial que empleaba precisamente una ocasión como esta para describir a los cretenses como mentirosos». Véase también Dobesch (1962a: 111 ss.). 


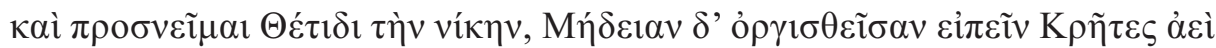

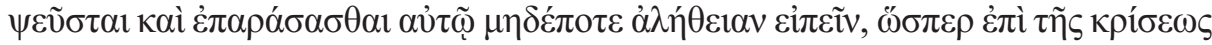

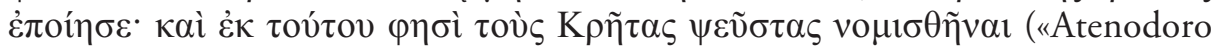
de Eretria, en el libro octavo de sus comentarios, dice que Tetis y Medea mantuvieron una disputa sobre quién era la más bella en Tesalia y el juez fue Idomeneo, quien otorgó la victoria a Tetis; Medea se irritó y dijo que los cretenses eran siempre unos mentirosos y lo maldijo con que nunca dijera la verdad, como había hecho en el juicio. Y a partir de ese hecho afirma que los cretenses son considerados mentirosos») $)^{4}$.

Evidentemente, no es preciso recurrir al mito para encontrar la causa de este uso negativo del verbo $\kappa \rho \eta \tau i \zeta \varepsilon v$. Tristemente para quienes tanto hemos disfrutado y disfrutamos de Creta, la mala fama de los cretenses como gente tramposa y engañadora estuvo muy extendida en la Antigüedad' y justifica por sí sola que 'comportarse

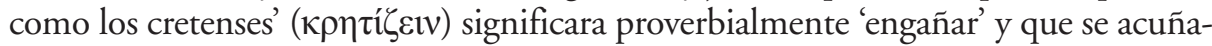
ran también otras varias expresiones proverbiales en las que el cretense aparece como prototipo del taimado engañador.

Así, Polibio en el libro octavo cuenta con toda clase de pormenores la historia del cretense Bolis, de quien ya en 8.16.4 se dice que «como cretense, era versátil por naturaleza" (ö $\tau \varepsilon$ K con Sosibio a ayudarle a salvar a Aqueo de caer en manos de Antíoco durante el asedio de Sardes, para lo cual se puso en contacto con otro cretense, su amigo y pariente Cámbilo, comandante de los soldados cretenses de Antíoco. Cuenta Polibio que Bolis y Cámbilo se reunieron en secreto y que «discutieron el asunto con visión cretense»

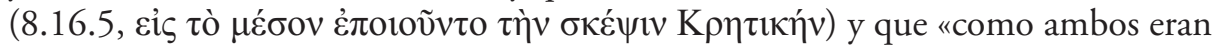
cretenses, necesitaron pocas palabras para llegar a la misma idea, que consistía en repartirse a partes iguales los diez talentos adelantados por Sosibio y delatar la empresa a Antíoco, y colaborar con él ofreciéndole poner a Aqueo en sus manos a cambio de dinero» (8.16.7-8). Puesto en marcha el plan, en la noche prevista Bolis se reúne con Aqueo para supuestamente ayudarle a escapar; Aqueo toma sus precauciones pero termina por fiarse de Bolis, en mala hora, porque, comenta Polibio $(8.19 .5)^{6}$, «desconocía lo del dicho, que se estaba comportando como un cretense con un cretense, y Bolis, en efecto, no había andado a tientas en ningún plan que pudiera servirle

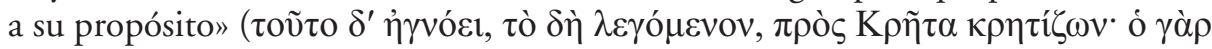

${ }^{4}$ Véase Nikolaidis, 1989: 400; Valverde Sánchez, 2016: 27 n. 16.

${ }_{5}^{5}$ Epiménides, fr. 1 Diels-Kranz; Calímaco, Himnos 1.8 Pfeiffer; Plutarco, Filopemen 13.9 ( «en Creta combatía al lado de los de Gortina, no como un hombre peloponesio y arcadio en una guerra franca y noble, sino adoptando la costumbre cretense, con sus trampas, engaños, raterías y asechanzas») y Emilio Paulo 26.2; Nuevo Testamento, Epistola a Tito 1.12, y otros textos recogidos por Goebel (1915: 77-81), Nikolaidis (1989), Spyridonidou-Skarsouli (1995: 387), Orth (2013: 377), Tronci (2015: 151-152) y Tosi (2017: no 349).

${ }^{6}$ Cf. Wunderer, 1898: 111. 


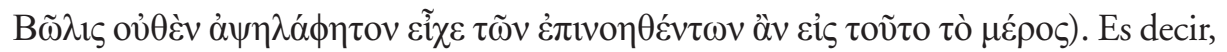
si precavido era Aqueo, más astuto aún era el cretense y, en efecto, la traición acaba consumándose.

Además de en Polibio, la expresión proverbial «comportarse como un cretense

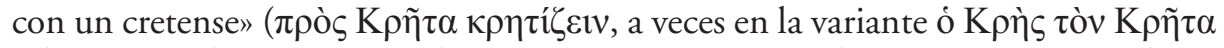
"el cretense al cretense») se documenta en otros textos literarios y es recogida en las colecciones paremiográficas y lexicográficas ${ }^{7}$, que son unánimes en la explicación

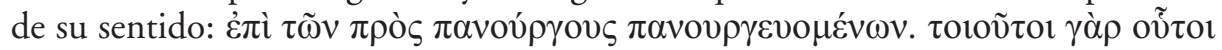
$\pi \alpha ́ v \tau \varepsilon \zeta$ («a propósito de quienes se comportan como bribones con bribones; porque así son todos esos»), según la glosa de la colección Zenobius Athous, que presenta

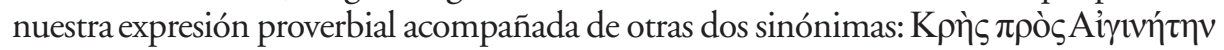

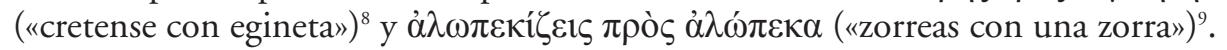

El sentido que las fuentes eruditas señalan para la expresión es corroborado por los testimonios literarios, donde se emplea dentro de un contexto ${ }^{10}$. Además de en Polibio, «comportarse como un cretense con un cretense» se documenta igualmente, entre otros autores, en Plutarco, quien emplea dos veces el proverbio. En una de ellas (Vida de Emilio Paulo 23.10-11) el contexto es comparable al de Polibio, porque hay cretenses de por medio y el proverbio se usa entonces semiliteralmente: el rey Perseo intenta engañar a sus soldados cretenses tratando de convencerlos, "entre súplicas y lágrimas», de que le devuelvan a cambio de dinero algunos objetos de oro de los que les había permitido apoderarse, y «entonces, a los que lo conocían bien, no les pasó desapercibido que se comportaba como un cretense con los cretenses»

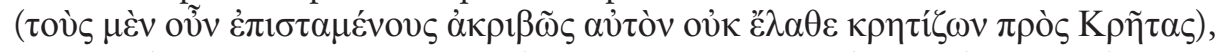
intentando engañar astutamente a los astutos cretenses. El segundo pasaje deja aún menos lugar a dudas sobre el carácter proverbial de la expresión, ya que es introducida

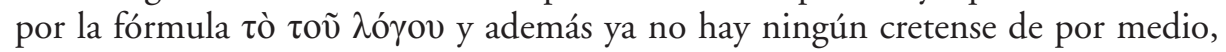
sino que quienes disputan en astucia y doblez son un espartano (Lisandro) y un persa (Farnabazo). Se trata de un pasaje de la Vida de Lisandro (20.2): $\pi \rho o ̀ \varsigma ~ K \rho \tilde{\eta} \tau \alpha ~ \delta \dot{~} \alpha$ ó $\rho \alpha$,

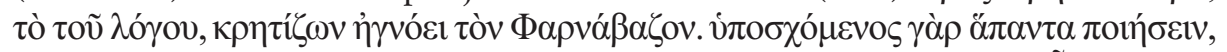

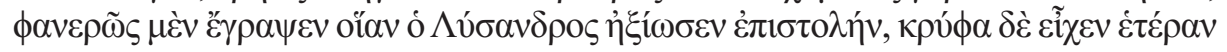

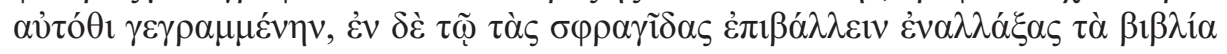

7 Zenobius Athous 5.53 (editado por Spyridonidou-Skarsouli), Collectio Bodleiana 794 (Gaisford 97), Diogeniano 7.31 y 7.65 ( $C P G$ I 291 y 297), Apostolio 12.61 y 14.98 ( $C P G$ II 557 y 628), Macario 7.35 (CPG II 205), D3 587 Cohn, Suda $\pi 2717$ y 2745 у к 2407 Adler, Erasmo 126 y 129, etc. Una excelente recopilación y estudio de los testimonios puede verse en Spyridonidou-Skarsouli (1995: 384-390).

${ }^{8}$ Se recoge también en Diogeniano 5.92 (CPG I 268), Macario 5.28 (CPG II 181), Apostolio 10.6 ( $C P G$ 487), Erasmo 127.

${ }^{9}$ Recogido también en Zenobius Vulgatus 1.70 (CPG I 25), Collectio Bodleiana 98 (Gaisford 10), Diogeniano 2.17 ( $C P G$ I 198), Gregorio de Chipre 1.40 (CPG I 352), etc.

${ }^{10}$ Véase Spyridonidou-Skarsouli (1995: 388-389). 


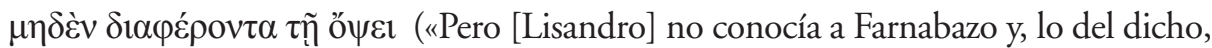
estaba haciendo el cretense con un cretense. Farnabazo, en efecto, le prometió hacer todo lo que le pedía y en su presencia escribió una carta en los términos que Lisandro le había reclamado, pero allí mismo tenía escondida otra ya escrita, y en el momento de poner los sellos cambió los rollos, que no se diferenciaban en nada a simple vista»).

La mala fama de los cretenses como prototipo de gentes engañosas, arteras y disimuladas está también presente en otra expresión proverbial que, al igual que «hacer el cretense con un cretense», suele emplearse en sentido negativo; me refiero

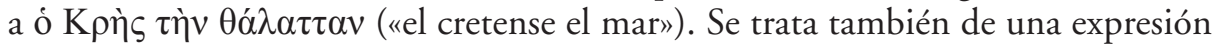
proverbial con frecuencia recogida y explicada por nuestras fuentes paremiográficas y lexicográficas ${ }^{11}$; en concreto, las colecciones Zenobius Vulgatus 5.30 y Zenobius

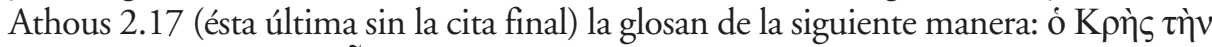

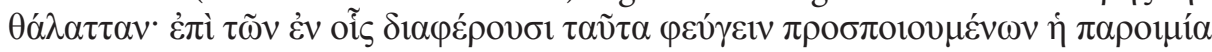

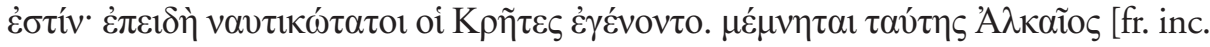
15 Voigt] (“"El cretense el mar”: El proverbio se dice de quienes fingen evitar aquello en lo que sobresalen. Porque los cretenses fueron muy marineros. Lo menciona Alceo»). Explicaciones semejantes se encuentran en otras recopilaciones, como Diogeniano Vindobonensis 3.41 (CPGII 42), Collectio Bodleiana 702 (Gaisford 85), D3 537 Cohn, Suda o 120 Adler. Por su parte, otras fuentes eruditas presentan una glosa algo más extensa, atribuyendo a la expresión proverbial el mismo sentido que los testimonios citados hasta aquí. Así, los escolios a Elio Aristides, Discursos 3.82-83 (A Platón, en defensa de los cuatro), glosan de la siguiente manera la expresión, citada por Elio Aristi-

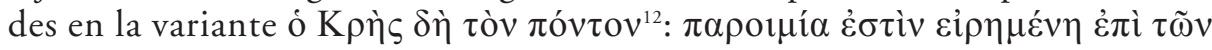

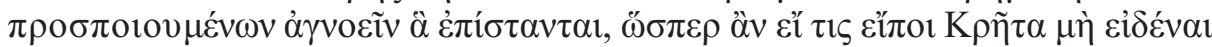

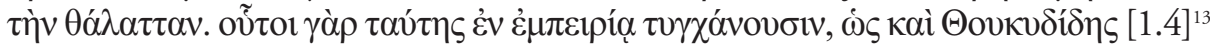

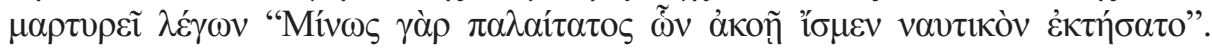

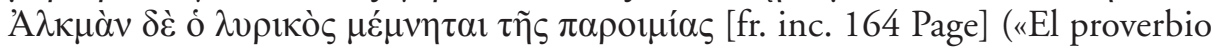
se dice a propósito de quienes fingen ignorar las cosas que saben, como si se dijera que un cretense no conoce el mar. Porque estos tienen experiencia en él, como también testimonia Tucídides cuando dice "pues Minos es el más antiguo de los que hemos oído decir que poseyó una flota”. Alcmán el poeta lírico menciona el proverbio»). Y Estrabón (10.4.17), basándose en Éforo (FgrH 70 F 149) ${ }^{14}$, reproduce prácticamente tal cual la explicación de paremiógrafos y lexicógrafos cuando señala que «los cretenses eran antaño dueños del mar, hasta el punto de que incluso "el cretense

${ }^{11}$ Una completa recopilación y estudio de conjunto de los testimonios puede verse en Bühler (1982 ss.: IV 148-153).

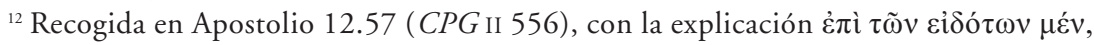

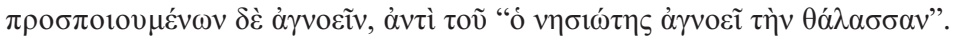

${ }^{13}$ Para otros testimonios sobre la pericia marinera de los cretenses, véase Bühler (1982 ss.: IV 150).

${ }^{14}$ Véase Keim (1909: 24); Dueck (2004: 43-44). 
el mar" se dice proverbialmente de quienes fingen ignorar las cosas que conocen

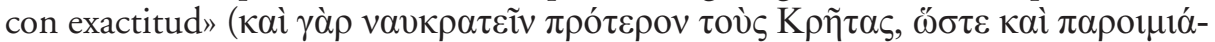

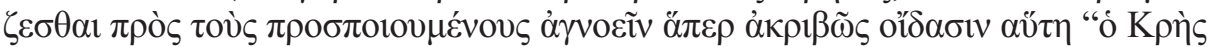

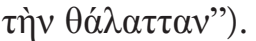

Únicamente difiere la explicación ofrecida en el léxico de Hesiquio (o 493

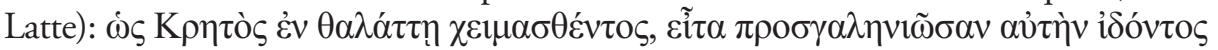

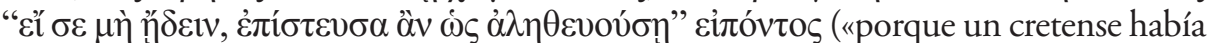
sido víctima de una tormenta en el mar y luego, cuando lo vio en calma, dijo: "si no te hubiera conocido, habría creído que eres sincero"»). Bühler (1982 ss.: IV 150) conjetura, y creo que tiene razón, que esta explicación tan diferente de la transmitida por el resto de las fuentes se debe a un error en la transmisión del texto (de un tipo frecuentísimo en la historia del texto de las colecciones paremiográficas) ${ }^{15}$, que ha acabado por trasladar a este proverbio la explicación que en realidad corresponde a otra expresión proverbial, «el siciliano el mar» (se diría a propósito de quienes, una vez tenida la experiencia de un peligro, no caen en el mismo error), que sigue a «el cretense el mar» en las colecciones alfabéticas de proverbios (Zenobius Athous 2.18) ${ }^{16}$.

Así pues, de acuerdo con la explicación casi unánime de las fuentes eruditas, la expresión proverbial «el cretense el mar» se diría irónicamente de quienes, por disimulo e interés, fingen ignorar ${ }^{17}$ aquello que conocen muy bien, de modo que también presentaría a los cretenses como gente engañosa y artera. Casi todos los textos literarios que documentan la expresión dentro de un contexto confirman que, en efecto, se empleaba con ese sentido negativo. Lo tiene en el Discurso 3 (82-83) de Elio Aristides, en el que el orador reprocha a Platón el haber ofrecido en sus diálogos una imagen negativa de Pericles, cuando conocía perfectamente sus cualidades, haciendo, pues, como el cretense que finge ignorar las cosas de la mar que tan bien conoce: $\check{\sigma} \sigma \pi \varepsilon \rho$

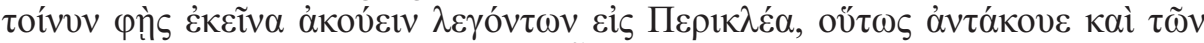

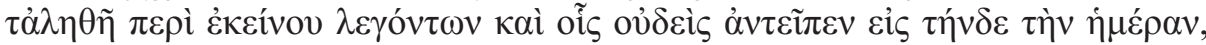

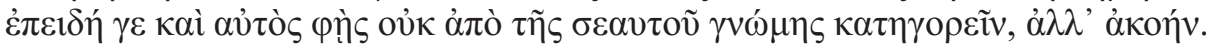

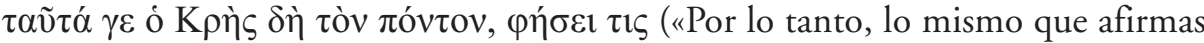
que has oído decir esas cosas [negativas] con respecto a Pericles, escucha a su vez también a quienes dicen la verdad sobre él y a quienes nadie ha contradicho hasta el día de hoy, dado que incluso tú mismo afirmas que no le acusas por propio conocimiento, sino de oídas. Eso, dirá alguno, es precisamente lo del cretense el mar»).

También a una ignorancia fingida hace referencia mediante el proverbio Aristéneto en una de sus Cartas (2.18), hablando de una mujer llamada Telxíona que «dejaba caer el velo sobre sus ojos para parecer casta, lo que le permitía calcular

${ }^{15}$ Véase García Romero (2020).

${ }^{16}$ Véase la recopilación de testimonios y el comentario de Bühler (1982 ss.: IV 154-159).

${ }^{17}$ En la breve Collectio Monacensis, inédita, el lema es precisamente, de acuerdo con la infor-

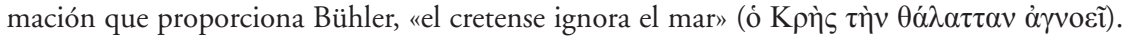


dónde dirigía sus furtivas miradas y corromper a los jóvenes sin quedar en evidencia». Telxíona hace creer a su joven admirador Pánfilo que es inexperta en las cosas del amor y que se ha enamorado por primera vez: «también el cretense parecía desconocer

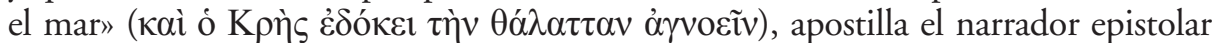
de la historia.

Un caso más complejo de uso de nuestra expresión proverbial es el que proporciona Libanio al comienzo de su epístola 491, datada en el año 356. Libanio recuerda a su corresponsal Barbación el hecho de que le había escrito anteriormente una carta que le fue entregada por Clemacio, «un hombre que ha pasado aquí todo el verano y todo el tiempo se lo pasó hablando de ti: estaba enseñando a quienes no ignoran,

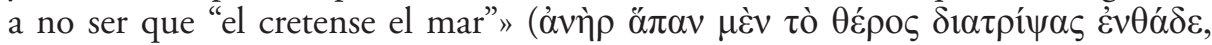

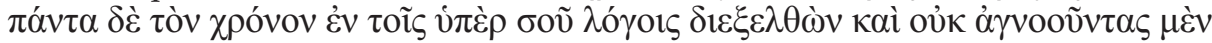

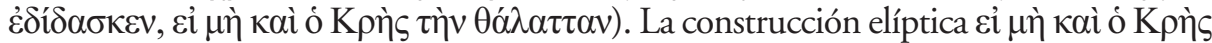

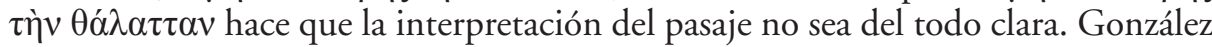
Gálvez (2005: 503) traduce: «sin embargo, estaba instruyendo a quienes no lo ignoraban, si no es cierto que "el cretense conoce el mar" ", y apunta en nota que «el sentido del proverbio es evidente y equivale al de llevar lechuzas a Atenas». De acuerdo con esta interpretación, Libanio estaría usando la expresión proverbial «el cretense el mar» en un sentido diferente del habitual, ya que no lo estaría empleando en sentido negativo, dicho de quienes fingen arteramente ignorar lo que conocen, sino en sentido literal para indicar que alguien dice algo a quienes conocen muy bien el tema, como los cretenses conocen muy bien el mar. Sería, entonces, una expresión proverbial sinónima de la mucho más habitual «lechuzas a Atenas», utilizada para hacer referencia a una acción inútil e incluso absurda como era llevar a un lugar algo que abundaba allí, y que el propio Libanio utiliza en otras de sus Cartas $(1262.1,1443.1)$ y se encuentra documentada ya en Aristófanes (Aves 301) ${ }^{18}$. Por su parte Goebel (1915: 80) opina que Libanio utiliza la expresión para referirse a algo que es imposible, como es imposible que un cretense desconozca la mar. En mi opinión, cabría otra interpretación que permitiría mantener en este contexto un sentido más cercano al habitual para «el cretense el mar», con el que estaría jugando irónicamente el orador: Clemacio estuvo hablando maravillas de ti, Barbación, diciendo cosas que a nosotros no nos

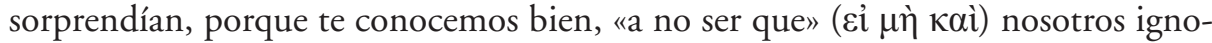
remos lo que creemos conocer bien, como el cretense finge que ignora el mar.

${ }^{18}$ Es un proverbio sobre el que tenemos abundantísimos testimonios, tanto en fuentes eruditas como en textos literarios. Lo recogen y comentan numerosas colecciones paremiográficas y lexicográficas y otras fuentes eruditas: Zenobius Vulgatus 3.6 (CPG I 59), Zenobius Athous 2.12 (Bühler, 1982 ss.: IV 114-122), Diogeniano 3.57 y 3.81 (CPG I 225 y 229), Collectio Bodleiana 276 (Gaisford 29), Apostolio V 46 ( $C P G$ II 345), Erasmo 2082; Focio $\gamma 126$ Theodoridis, Suda 1336 Adler; escolios a Aristófanes, Caballeros 1093 y a Aves 301; Eustacio, Comentario a la Ilíada 87, 44 ss. Se encuentra también bien documentado en textos literarios: Demón, FgrH 327 F 15; Luciano, Nigrino, carta introductoria; Diógenes Laercio 3.47; Cicerón, Cartas a allegados 9.3 y 6.3, Cartas a su hermano Quinto 2.15. Véase Bühler (1982 ss.: IV 114-122), Menor Martínez (2009) y Tosi (2017: no 584). 
La mala fama proverbial de los cretenses ha podido provocar incluso que se interpretara en sentido negativo un proverbio que probablemente no lo tenía en origen:

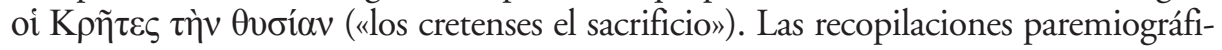
cas más importantes, como Zenobius Vulgatus 5.50 (CPG I 141) y Zenobius Athous

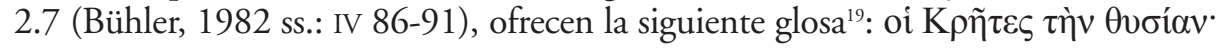

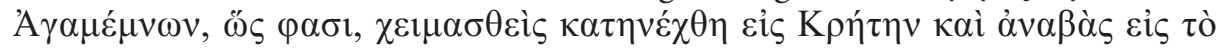

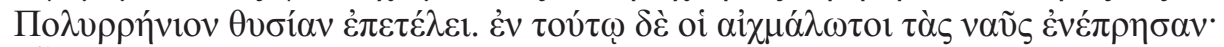

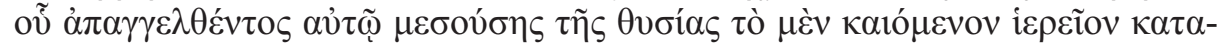

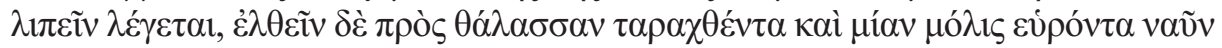

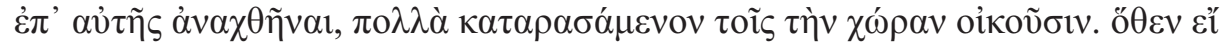

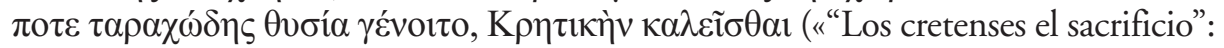
Cuentan que Agamenón fue llevado por una tempestad a Creta, y subió al Polirrenio ${ }^{20}$ e hizo un sacrificio. Estando en ello, los prisioneros de guerra quemaron las naves, y se dice que, cuando se lo anunciaron a la mitad del sacrificio, abandonó a la víctima que se estaba quemando y alborotado marchó hacia el mar; y hallando apenas una sola nave zarpó en ella, mientras lanzaba muchas maldiciones contra los habitantes del lugar. Por lo cual, cuando se produce un sacrificio alborotado, se denomina "cretense" )).

Aunque diversas fuentes ${ }^{21}$ comentan el arribo de Agamenón a Creta a causa de una tormenta, durante su viaje de regreso de Troya, ninguna de ellas se refiere al sacrificio interrumpido que relatan las colecciones paremiográficas. Por otro lado, como han estudiado muy bien Dobesch y Bühler, la explicación de los paremiógrafos presenta puntos confusos, sobre todo el hecho de que, tal como se narran los hechos, no se entiende por qué Agamenón se indigna contra los cretenses y se marcha lanzando maldiciones contra ellos, cuando se ha dicho que los culpables

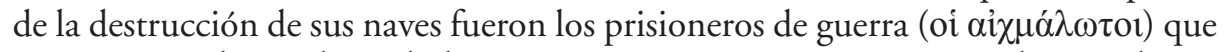
traía consigo, los cuales todo hace suponer que eran troyanos ${ }^{22}$. Los dos estudiosos citados sugieren, convincentemente en mi opinión, que la expresión proverbial «los cretenses el sacrificio» no tenía originalmente ninguna relación con el relato del sacrificio supuestamente perturbado de Agamenón, que sería una explicación

${ }^{19}$ Explicaciones similares se encuentran en Suda or 83 Adler y Collectio Bodleiana 719 (Gaisford 87) y, de forma muy abreviada, en Diogeniano 7.5 (CPG I 287) y Apostolio 12.41 (CPG II 552). dos trabajos.

${ }^{20}$ Un lugar, por cierto, a cuyas inscripciones Ángel Martínez Fernández ha dedicado estupen-

${ }^{21}$ Veleyo Patérculo 1.1.2; Esteban de Bizancio $410.5=\lambda 31$ Billerbeck; Diodoro 33.11; Servio, ad Aen. 3.133. Cf. Dobesch (1962a: 90 ss.) y Bühler (1982 ss.: IV 87-88).

${ }^{22}$ Las fuentes que hablan del paso de Agamenón por Creta comentan sobre todo que fundó en la isla algunas ciudades, y algunas de esas fuentes hacen referencia a maldiciones lanzadas por Agame-

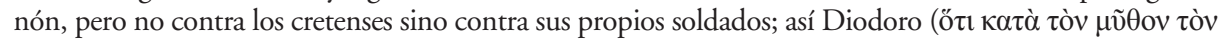

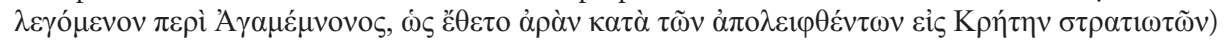
y Servio (desertores). 
añadida secundariamente; su origen habría que buscarlo más bien en alguna característica especial que tendría algún sacrificio llevado a cabo por los cretenses, de donde habría nacido la expresión proverbial para indicar un acto de culto interrumpido y perturbado (aunque lo cierto es que no podemos estar absolutamente seguros de que ese fuera el sentido del proverbio, ya que no contamos con ningún testimonio de uso en el que aparezca empleado dentro de un contexto).

En concreto, Bühler piensa en un tipo de ritual semejante a aquellos en los que el sacerdote encargado de hacer el sacrificio huye tras haberlo llevado a cabo, como sucedía en las Bufonias atenienses, de acuerdo con la noticia transmitida por Pausanias 1.24.4 («Llaman 'matabueyes' a uno de los sacerdotes, el cual, tras matar al buey y arrojar el hacha allí -pues así es el rito-, se marcha huyendo. Y ellos, como no saben quién ha llevado a cabo la acción, someten al hacha a un juicio»), o en el caso de la ternera recién nacida que los habitantes de Ténedos sacrificaban a Dioniso, según el relato de Eliano, Naturaleza de los animales 12.34 («el que le asesta el golpe con el hacha es apedreado por la gente y escapa hasta el mar»). Pueden citarse, en efecto, varias expresiones proverbiales comparables que, de acuerdo con las explicaciones transmitidas por paremiógrafos y lexicógrafos, se habrían originado a partir de sacri-

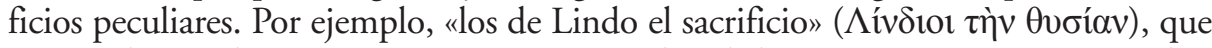
en Zenobius Vulgatus 4.95 (CPGI 113) se explica de la siguiente manera ${ }^{23}$ : «Proverbio a propósito de quienes realizan actos sagrados pronunciando malas palabras. A partir de Heracles, que en Lindo arrebató un buey a un campesino y celebró un banquete, mientras el campesino lo insultaba. Por eso los de Lindo, de acuerdo con un oráculo, realizan sacrificios a Heracles así, insultándolo». O, también con Agamenón como

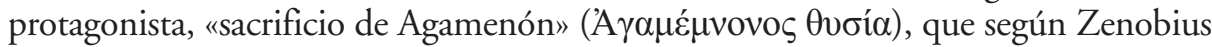
Vulgatus 1.13 (CPG I 4) se decía «a propósito de los que son difíciles de persuadir y tozudos. Porque cuando Agamenón estaba haciendo un sacrificio en Troya, el buey huyó y a duras penas lo capturaron y lo trajeron $»^{24}$.

${ }^{23}$ Se encuentra recogido también en Collectio Bodleiana 607 (Gaisford 72), Diogeniano 6.15 (CPG I 272), Apostolio 10.71 (CPG II 506) y Hesiquio $\lambda 1061$ Latte, y la historia era narrada en los Aitia de Calímaco (fr.22-23 Pfeiffer) y otras fuentes citadas por Lelli et alii (2006: $441 \mathrm{n} .441$ );

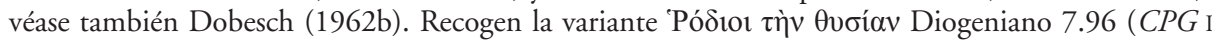
303), Gregorio de Chipre 3.57 (CPG I 372), Apostolio 15.29 (CPG II 635) y Hesiquio $\rho 399$ Latte.

${ }^{24}$ También en Collectio Bodleiana 5 (Gaisford 1), Diogeniano 1.6 (CPGI 181), Apostolio 1.26 ( $C P G$ II 245) y Macario 1.9 ( $C P G$ II 136); este último atribuye la explicación a Demón (FgrH 327 F 9).

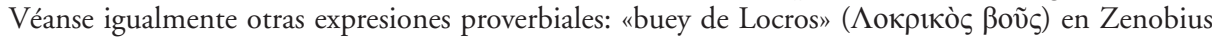
Vulgatus 5.5 (CPG I 116): «el proverbio se aplica a propósito de las cosas baratas. Porque los locrios, una vez que no disponían de un buey para un sacrificio costeado a expensas públicas, colocaron unos maderos pequeños como apoyo para unos pepinos e hicieron la figura de un buey, y así rindieron culto

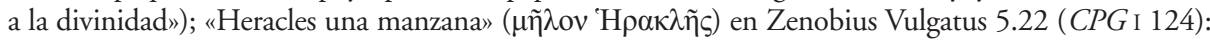
"Apolodoro, en su Sobre los dioses [FgrH 244 F 145], afirma que en Atenas se hace un sacrificio singular en honor de Heracles Alejador de Males. Pues una vez que se escapó un buey que iba a ofrecerse a Heracles, cogieron una manzana, le pusieron cuatro ramas a modo de patas y dos a modo de cuernos 
En lugar de una glosa de ese tipo que relacionaría el origen de la expresión proverbial «el cretense el mar» con algún peculiar sacrificio cretense, en nuestras recopilaciones paremiográficas y lexicográficas ha prevalecido una explicación falsa en la que, una vez más, los cretenses desempeñan el papel de malvados, en este caso sin venir a cuento. Posiblemente la mala fama proverbial de los cretenses (qui saepissime vituperantur, en palabras de Goebel [1915: 77]) ha podido influir en el predominio de esa falsa explicación. No en vano el léxico bizantino Suda, una de las fuentes que se refiere al sacrificio frustrado de Agamenón como origen del proverbio "el cretense el mar",

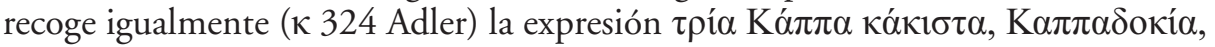

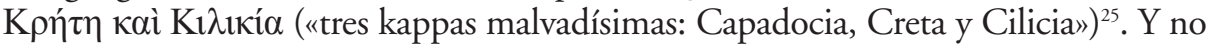
en vano la ya mala fama proverbial de los cretenses en el mundo pagano perduró en el mundo cristiano, con la ayuda de la cita de la famosa paradoja de Epiménides (despojada de su esencial carácter paradójico) en la paulina Epistola a Tito (1.12): «Dijo uno de ellos, su propio profeta: "cretenses siempre mentirosos, malas bestias, barrigas ociosas"». Un Epiménides que, por cierto, también entró a formar parte del refranero griego antiguo, en dos expresiones proverbiales: «sueño de Epiménides»

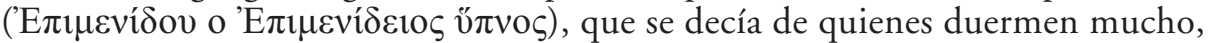
a partir de la tradición que atribuía a Epiménides un sueño de años ${ }^{26}$; $\mathrm{y}$ «la piel de

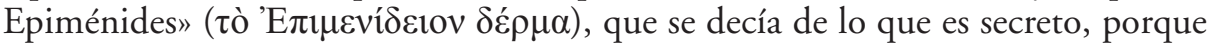
se contaba que los espartanos conservaban la piel del sabio cretense, toda ella tatuada con signos secretos ${ }^{27}$.

Deseo de corazón a mi querido Ángel que siga haciendo felizmente el cretense (en sentido literal) durante muchos años y mostrando que la $\mathrm{K}$ de Creta (como la de Canarias), lejos de ser "malvadísima", está repleta de gentes estupendas.

\section{REFERENCIAS BIBLIOGRÁFICAS}

Amado Rodríguez, María Teresa (1995): «Verbos denominativos derivados de gentilicios y topónimos», Myrtia 10: 67-103.

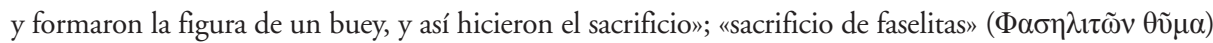
en Zenobius Vulgatus 6.36 (CPG I 172): «se dice a propósito de los sacrificios de poco valor e incruentos. Porque afirman que los habitantes de Faselis ofrecen salazones como sacrificio a los dioses»; «sacrificio cario» (Kapıkòv $\theta \tilde{u} \mu \alpha)$ en Zenobius Athous 3.104 (Miller 373) = Plutarco 1.73 (CPG I 332): «a propósito de quienes ofrecen en sacrificio miembros sin carne. Porque los carios sacrifican perros».

${ }^{25}$ Erasmo 2582. Nikolaidis (1989: 402) indica que ha perdurado en griego moderno, en la variante "Creta, Chipre y Cefalonia».

${ }^{26}$ Zenobius Athous 1.20 (Miller 351), Gregorio de Chipre 2.23 (CPG I 360), Collectio Coisliniana 209 (Gaisford 139), Diogeniano Vindobonensis 2.49 (CPG II 25). Véase Luciano, Timón 6.

${ }^{27}$ Zenobius Athous 2.72 (Bühler, 1982 ss.: v 324-334, donde puede encontrarse un completísimo estudio), Collectio Bodleiana 875 (Gaisford 107), Collectio Coisliniana 209 (Gaisford 139), Diogeniano 8.28 (CPG I 309), Apostolio 17.14 (CPG II 689), Suda \& 2471 Adler. 
BÜHLER, Winfred (1982 ss.): Zenobii Athoi proverbia (I: Prolegomena; IV: Libri secundi proverbia 1-40; V: Libri secundi proverbia 41-108), Vandenhoeck \& Ruprecht, Göttingen.

CoHN, Leopold (1891): «Zur Überlieferung des alphabetischen Corpus», en O. CRUsIUs - L. CoHN, Zur handschriftlichen Überlieferung, Kritik und Quellenkunde der Paroemiographen; mit einem Anhange: Die Sprichwörter des Eustathios, von E. Kurtz, Dieterich, Göttingen, pp. 224-267 [recogido en Supplementum ad Paroemiographos Graecos, Olms, Hildesheim 1961, IV].

DoBESCH, Gerhardt (1962a): Die Sprichwörter der griechischen Sagengeschichte, Viena [Tesis Doctoral].

DobesCH, Gerhardt (1962b): «Studien zu Sprichwörtern», Wiener Studien 75: 94-99.

DUECK, Daniela (2004): «"Bird's milk in Samos”. Strabo's use of geographical proverbs and proverbial expressions», Scripta Classica Israelica 23: 41-56.

ERASMO = ÉRASME DE ROTTERDAM (2011): Les Adages d'Érasme, latin-français, sous la direction de J.-Ch. Saladin, Les Belles Lettres, París.

GaIsford, Thomas (1836): Paroemiographi Graeci, Oxford University Press, Oxford [reimpr. Biblio Verlag, Osnabrück, 1972].

García Romero, Fernando (2020): «La paremiografía y la lexicografía como fuente de la literatura griega», en El foro de los clásicos. Actas del XV Congreso Español de Estudios Clásicos, Sociedad Española de Estudios Clásicos, Madrid [en prensa].

GoEBEL, Maximilian (1915): Ethnika. De Graecarum civitatum proprietatibus proverbio notatis, A. Favorke, Bratislava.

GonZÁlez GÁlvez, Ángel (2005): Libanio: Cartas, libros I-V, Gredos, Madrid.

KEIM, Joseph (1909): Sprichwörter und paroemiographische Überlieferung bei Strabo, H. Laupp, Tübingen.

LeLli, Emanuele (et al.) (2006): I proverbi greci. Le raccolte di Zenobio e Diogeniano, Rubbettino, Soveria Mannelli.

LEUTSCH, Ernst Ludwig VON - SCHNEIDEWIN, Friedrich Wilhelm (1839-1851): Corpus Paroemiographorum Graecorum, Vandenhoeck \& Ruprecht, Göttingen [reimpr. Olms, Hildesheim 1965].

Mariño Sánchez-Elvira, Rosa María - García Romero, Fernando (1999): Proverbios griegos. Menandro: Sentencias, Gredos, Madrid.

Menor Martínez, Mónica (2009): «Llevar lechuzas a Atenas no vale un óbolo», Paremia 18: 217-224.

MilLER, Emanuel (1868): Mélanges de littérature grecque, L’Imprimerie Impériale, París [reimpr. Hakkert, Amsterdam 1965], pp. 341-384.

Movellán Luis, Mireia (2015): La crónica troyana de Dictis de Creta, Madrid [Tesis Doctoral de la Universidad Complutense (https://eprints.ucm.es/33227/1/T36401.pdf)].

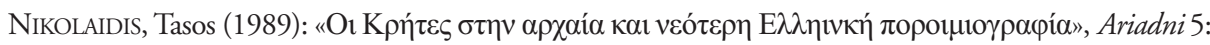
$399-406$.

OrTH, Christian (2013): Alcaeus, Amipsias, Apollophanes, Verlag Antike, Heidelberg.

SPYRIDONIDOU-SKarsouli, María (1995): Der esrte Teil der fünften Athos-Sammlung griechischer Sprichwörter, De Gruyter, Berlín-Nueva York.

Tosi, Renzo (2017): Dizionario delle sentenze latine e greche, Rizzoli, Milán.

TRONCI, Liana (2015): «Verbi in -í̧ etnonimici nella storia del greco (e oltre). Lingua e costruzione di identità», en M. BENEDETTI (ed.), Rappresentazioni linguistiche dell'identità, Università "L'Orientale", Nápoles, pp. 141-159. 
VAlverde SÁnchez, Mariano (2016): El mito de Idomeneo y su tradición literaria, Signifer Libros, Salamanca.

WUNDERER, Carl (1898): Polybios-Forschungen. I: Sprichwörter und sprichwörtliche Redensarten bei Polybios, Dieterich, Leipzig [reimpr. SDZ, Aalen 1969]. 